\title{
Dual Linearised Gravity in Arbitrary Dimensions
}

\author{
K M Ajith ${ }^{\dagger}$, E Harikumar ${ }^{\ddagger}$ and M Sivakumar ${ }^{\dagger}$ \\ $\dagger$ School of Physics, University of Hyderabad, \\ Central University P. O, Hyderabad-500046, India \\ ¥ Instituto de Física, Universidade de São Paulo, \\ Caixa Postal 66318, CEP 05315-970, São Paulo - SP, Brazil \\ E-mail: ph01ph13@uohyd.ernet.in,hari@fma.if.usp.br and \\ mssp@uohyd.ernet.in
}

\begin{abstract}
We construct dual formulation of linearised gravity in first order tetrad formalism in arbitrary dimensions within the path integral framework following the standard duality algorithm making use of the global shift symmetry of the tetrad field. The dual partition function is in terms of the (mixed symmetric) tensor field $\Phi_{\left[\nu_{1} \nu_{2} \cdots \nu_{d-3}\right] \nu}$ in frame-like formulation. We obtain in d-dimensions the dual Lagrangian in a closed form in terms of field strength of the dual frame-like field. Next by coupling a source with the (linear) Riemann tensor in d-dimensions, dual generating functional is obtained. Using this an operator mapping between (linear) Riemann tensor and Riemann tensor corresponding to the dual field is derived and we also discuss the exchange of equations of motion and Bianchi identity.
\end{abstract}

PACS numbers: $4.50+\mathrm{h}, 11.90+\mathrm{t}, 11.25 \mathrm{Tq}, 11.15 .-\mathrm{q}$

Keywords: Duality, Spin-2 theory, Mixed symmetric tensor gauge field 


\section{Introduction}

The construction and study of dual formulation of physical systems have been of interest always[1]. The different but equivalent formulation of a system is useful for studying different phases or approximation that may not be transparent in one description. Among the dual formulations in d-dimensions, duality between scalar field and $d-2$ form theories is well-known. Currently there are many interesting attempts to extend this duality to spin-2 theory (linear gravity) in particular and to arbitrary spin-s theories in general. Part of the motivation for the former is to understand hidden symmetry in gravitational theory, if any. An earliest approach to obtain strong-weak duality for gravitational theory was first made in [2]. An analogue of $\mathrm{S}$ duality for $4 \mathrm{~d}$ linearised gravity in MacDowell-Mansouri formalism was made in [3]. It has been shown recently in $[4,5,6,7,8]$ that the spin-2 theory can be formulated in terms of a mixed symmetric tensor having Young symmetry $(\mathrm{d}-3,1)$ in d-dimensions. This was obtained either as on-shell equivalent theories or in a non-covariant way. Mixed symmetric tensor gauge fields have been studied, more as a generalisation of $p$-form theories $[9,10]$. Curtright [10] in particular has given a construction in terms of $\phi_{[\mu \nu] \lambda}$ with $\phi_{[[\mu \nu] \lambda]}=0$ (here, square bracket [] denotes antisymmetry), which describes linearised gravity in 5-dim. Zinoviev [11] has generalised this construction in a first order formulation for more general cases. Recently, starting from a first order formulation of spin-2 theory [12], Boulanger, Cnockaert and Henneaux [13] have constructed dual spin-2 theory in a manifestly covariant and off-shell formulation. These authors have shown that in 4dimensions, the dual theory is same as the original Fierz-Pauli (FP)theory while in 5-dimensions, the dual theory turned out to be the one given by Curtright[10]. It has been also shown that in arbitrary d-dimensions, the dual theory is formulated in terms of field, $\phi_{\left[\mu_{1} \mu_{2} \cdots \mu_{d-3}\right] \nu}$ obeying the condition of vanishing cyclic sum of indices, i.e., $\phi_{\left[\left[\mu_{1} \mu_{2} \cdots \mu_{d-3}\right] \nu\right]}=0$. This construction has been also been obtained starting from AdS background by Matveev and Vasiliev [14]. The duality invariance of the 4d linearised gravity action was shown by Henneaux and Teitelboim [15]. There are also attempts to extend dual construction to non-linear gravity also [16]. All these studies have been made in metric-like formulation. In this work we construct dual theory for $\mathrm{s}=2$ in frame-like formulation.

Dual theories have been generally obtained based on the algorithm developed in [17]. This method relies on the existence of a global symmetry and has been fruitfully applied in string theory[18]. This method has also been applied to show Bosonisation as duality [19], to exhibit different formulations of massive gauge theories as dual formulations [20] and also in fractional quantum Hall effect [21] (for a review of this method, see [22]). This raises the question of deriving the dual spin-2 theories applying this method. In this paper we show that within this standard duality construction, based on the existence of the global shift symmetry, dual partition function and the generating functional for linear gravity in d-dimensions can be obtained in frame-like formulation

The standard FP theory (which is same as the linear level approximation of general 
relativity) has symmetric second rank tensor $h_{\mu \nu}$ as the basic field and its Lagrangian is

$$
L=\frac{1}{2} \partial_{\alpha} h^{\mu \nu} \partial^{\alpha} h_{\mu \nu}-\partial_{\nu} h^{\nu \mu} \partial^{\beta} h_{\beta \mu}+\partial_{\nu} h^{\nu \mu} \partial_{\mu} h_{\alpha}^{\alpha}-\frac{1}{2} \partial^{\mu} h_{\alpha}^{\alpha} \partial_{\mu} h_{\beta}^{\beta}
$$

which is invariant under $\delta h_{\mu \nu}=\partial_{\mu} \epsilon_{\nu}+\partial_{\nu} \epsilon_{\mu}$. Instead of the metric-like formulation, i.e., symmetric tensor, as the dynamical field, one can have an equivalent spin-2 description in terms of the frame field, i.e., tetrad field $e_{\mu \nu}$ (where no symmetry is assumed between the indices). The corresponding Lagrangian is [11]

$$
L=\frac{1}{8} T_{[\mu \nu] \lambda} T^{[\mu \nu] \lambda}+\frac{1}{4} T_{[\mu \nu] \lambda} T^{[\mu \lambda] \nu}-\frac{1}{2} T_{\mu} T^{\mu}
$$

where the field strength is $T_{[\alpha \beta] \lambda}=\partial_{\alpha} e_{\beta \lambda}-\partial_{\beta} e_{\alpha \lambda}$ and $T_{\alpha}=T_{[\alpha \beta]}^{\beta}$. Note that the world index and tangent space index are not distinguished since we work at the linear level. The above Lagrangian is trivially invariant under the transformation

$$
\delta_{\epsilon} e_{\mu \nu}=\partial_{\mu} \epsilon_{\nu}
$$

and for the specific choice of the coefficients in equation (2) under

$$
\delta_{\Lambda} e_{\mu \nu}=\Lambda_{[\mu \nu]} .
$$

Using this invariance in equation (2) one can gauge away the anti-symmetric part of the field $e_{\mu \nu}$ leaving only the symmetric part and then equation (2) reduces to Lagrangian in equation (1). The first order Lagrangian equivalent to the above one in equation (2) is given by

$$
L_{I}=-\frac{1}{2} \omega^{\mu[\nu \alpha]} \omega_{\nu[\mu \alpha]}-\frac{1}{2} \omega^{\mu[\nu \alpha]} T_{[\nu \alpha] \mu}+\frac{1}{2} \omega_{\mu} \omega^{\mu}-\omega^{\mu} T_{\mu} .
$$

Here $\omega_{\mu}=\omega_{\nu \mu}^{\nu}$. This Lagrangian is invariant under the transformations in equation(3) as well as under

$$
\delta_{\Lambda} \omega_{\mu[\alpha \beta]}=\partial_{\mu} \Lambda_{[\alpha \beta]}, \quad \delta_{\Lambda} e_{\mu \nu}=\Lambda_{[\mu \nu]} .
$$

Note that this first order form is valid in d-dimensions and differs from the starting point of [13] by a field redefinition. $\ddagger$ In this paper we start with the Lagrangian given in equation (5) instead of FP theory directly and obtain the equivalent dual model in arbitrary dimensions, but in frame-like formulation.

In the method adopted [17] one considers a theory which has a global symmetry and this global symmetry is gauged to a local one by introducing an appropriate gauge field. The dual field strength of the gauge field is then constrained to vanish by means of a multiplier field. Integrating the multiplier field and the gauge field (which will become pure gauge now), original theory results. Instead, integrating out the original field and the gauge field gives the dual theory where the multiplier field becomes the dynamical field. It is well known that this method applied to massless scalar field leads to d-2 form $\ddagger$ The field redefinition

$$
\omega_{\alpha[\beta \gamma]}=Y_{[\beta \gamma] \alpha}+\frac{2}{D-2} \eta_{\alpha[\beta} Y_{\gamma] \rho}^{\rho}
$$

in (5) leads to the starting point of [13] (up to an overall scale). 
gauge theory. Applied to Maxwell theory in 4-dimensions, lead again to Maxwell action in terms of dual vector potential[23].

The global symmetry which we make use of is shift of the tetrad field. Hence it is extendable to coupling sources with suitable fields, such that global symmetry is preserved. Then the equivalence between the correlation functions can be derived. The main results obtained here are :

(i) Derivation of the dual partition function for linear gravity, described by the dual frame-like field, $\Phi_{\left[\nu_{1} \nu_{2} \cdots \nu_{d-3}\right] \nu}$ in a closed form in terms of field strength.

(ii) By coupling source to (linear) Riemann tensor, we obtain the equivalent dual generating functional and infer the operator mapping between Riemann tensor and its dual equivalent.

This paper is organized as follows. In section 2 we consider 4-dim. spin-2 theory described by equation (5) and, derive equivalent theory. In section 3, deals with duality in dimensions $\mathrm{d}>4$. In Section 4 dualisation of the theory with external source is dealt and the mapping between the correlators are derived. Our concluding remarks are given in section 5. We use $\epsilon_{0,1,2 . . d-1}=1$ and the metric $g_{\mu \nu}=\operatorname{diag}(1,-1,-1,-1, \cdots,-1)$. Appendix gives the details of duality between the equation of motion and Bianchi identity.

\section{Dual theory in $3+1$-dimensions}

In this section we construct the dual formulation of massless spin- 2 theory described by the Lagrangian in equation (5) in $3+1 \mathrm{dim}$. The first-order theory described by the Lagrangian in equation (5) is invariant under the gauge transformations given in equation (6). Apart from this, the Lagrangian is also invariant under the shift of $e_{\mu \nu}$ field by a global parameter, i.e., under

$$
e_{\mu \nu} \rightarrow e_{\mu \nu}+\epsilon_{\mu \nu}
$$

We gauge this symmetry by introducing a gauge field $K_{[\mu \nu] \lambda}$ which transform as $K_{[\mu \nu] \lambda} \rightarrow K_{[\mu \nu] \lambda}+\partial_{\mu} \epsilon_{\nu \lambda}-\partial_{\nu} \epsilon_{\mu \lambda}$, there by making the theory invariant under local shift of $e_{\mu \nu}$ field. Here the gauge field $K_{[\mu \nu] \lambda}$ is a mixed symmetric tensor having same symmetry in its indices as the field $T_{[\mu \nu] \lambda}$. Now to bring it equivalent to original theory, the dual of the field strength $\partial_{\mu} K_{[\nu \sigma] \beta}$ should vanish. This is enforced by a multiplier

field $\phi_{\beta \rho}$ (there is no symmetry in the indices of multiplier field). Thus the master Lagrangian which is equivalent to equation (5) is :

$$
\begin{aligned}
L_{M}^{(4)} & =-\frac{1}{2} \omega^{\mu[\nu \alpha]} \omega_{\nu[\mu \alpha]}+\frac{1}{2} \omega^{\mu} \omega_{\mu}-\frac{1}{2} \omega^{\beta[\nu \sigma]}\left(T_{[\nu \sigma] \beta}-K_{[\nu \sigma] \beta}\right) \\
& -\omega^{\nu} T_{\nu}+\omega^{\nu} K_{\nu}+\frac{1}{4} \epsilon^{\mu \nu \sigma \rho}\left(\partial_{\mu} K_{[\nu \sigma] \beta}\right) \phi_{\rho}^{\beta} .
\end{aligned}
$$

This master Lagrangian has to satisfy two conditions: (1) It must possess all the symmetries of the original Lagrangian given in equation (6) and (2) when the multiplier field equation is imposed, the master Lagrangian should reduce to the original theory. 
The first condition is satisfied by demanding that under the transformation of $e_{\mu \nu}$ and $\omega_{\nu[\alpha \beta]}$ in equation (6), we also have

$$
\delta_{\Lambda} K_{[\nu \mu] \alpha}=0, \quad \delta_{\Lambda} \phi_{\mu \nu}=\epsilon_{\mu \nu \rho \sigma} \Lambda^{[\rho \sigma]} \equiv \tilde{\Lambda}_{[\mu \nu]}
$$

It is interesting to note that the transformation parameter of original field $e_{\mu \nu}$ and the multiplier field $\phi_{\mu \nu}$ are dually related, which means both undergo transformation by an antisymmetric parameter. The invariance under the transformation in equation (3) is trivially present as the fields $e_{\mu \nu}$ appear in $L_{M}^{(4)}$ only through $T_{[\mu \nu] \alpha}$. It is easy to see that the second condition is satisfied as eliminating $\phi_{\mu \nu}$ field sets $K_{[\mu \sigma] \beta}=\partial_{\mu} \epsilon_{\sigma \beta}-\partial_{\sigma} \epsilon_{\nu \beta}$ which can be used to redefine $e_{\mu \nu}^{\prime}=e_{\mu \nu}-\epsilon_{\mu \nu}$ to get back the original theory. Note that in the action $L_{M}, \phi_{\mu \nu}$ is arbitrary up to an addition of $\partial_{\mu} \tilde{\epsilon}_{\nu}$. This translates into gauge symmetry of the dual field.

We start the dualisation from the partition function

$$
Z=\int D \phi_{\alpha \beta} D e_{\mu \nu} D \omega_{\sigma[\rho \lambda]} D K_{[\theta \tau] \gamma} e^{-i \int d^{4} x L_{M}^{(4)}}
$$

where the Lagrangian is given in equation (9). Now to obtain the dual theory we integrate out the gauge field. As the gauge field appears only linearly in the action, integrating $K_{[\nu \sigma] \beta}$ in the above partition function results the delta function

$$
\delta\left(\frac{1}{2} \omega^{\beta[\nu \sigma]}+\frac{1}{2}\left(\omega^{\nu} g^{\sigma \beta}-\omega^{\sigma} g^{\nu \beta}\right)+\frac{1}{4} \epsilon^{\mu \nu \sigma \delta} \partial_{\mu} \phi_{\delta}^{\beta}\right)
$$

in the measure. The integration over $\omega$ field are now trivial as this delta function allows one to replace the $\omega$ fields in terms of the multiplier field as given below.

$$
\begin{aligned}
\omega^{\nu} & =\frac{1}{4} \epsilon^{\nu \mu \gamma \beta} F_{[\mu \gamma] \beta} \\
\omega^{\beta[\nu \sigma]} & \left.=\frac{1}{4}\left[\left(g^{\beta \sigma} \epsilon^{\nu \mu \gamma \lambda}-g^{\beta \nu} \epsilon^{\sigma \mu \gamma \lambda}\right) F_{[\mu \gamma] \lambda}-2 \epsilon^{\nu \sigma \mu \gamma} F_{[\mu \gamma]}{ }^{\beta}\right)\right]
\end{aligned}
$$

where $F_{[\mu \nu] \beta}=\partial_{\mu} \phi_{\nu \beta}-\partial_{\nu} \phi_{\mu \beta}$ is the field strength of multiplier field. It is easy to see that when these solutions for $\omega$ are plugged back in the action, all $e$ dependent terms vanish and then the integration over $e$ field just gives a multiplicative infinity which can be absorbed in the normalization. Thus the partition function corresponding to the dual theory is

$$
Z=\int D \phi_{\mu \nu} e^{-i \int d^{4} x L_{D}^{(4)}}
$$

where the effective Lagrangian is

$$
L_{D}^{(4)}=\frac{1}{8} F_{[\mu \gamma] \lambda} F^{[\mu \gamma] \lambda}+\frac{1}{4} F_{[\mu \gamma] \lambda} F^{[\mu \lambda] \gamma}-\frac{1}{2} F^{\mu} F_{\mu}
$$

where $F_{\mu}=F_{\mu \nu}{ }^{\nu}$. Thus we see that the dual description is in terms of non symmetric, rank -2 tensor. It is obvious that the above dual Lagrangian has $\delta \phi_{\mu \nu}=\partial_{\mu} \tilde{\epsilon}_{\nu}$ symmetry as $F_{[\mu \gamma] \lambda}$ has this symmetry. This Lagrangian is exactly the same in structure and coefficients as that in the equation (2). Hence the dual theory also has the same symmetry $\delta \phi_{\mu \nu}=\tilde{\Lambda}_{[\mu \nu]}(x)$. 
This result can also be seen directly by starting from the second order theory given in equation (2). The corresponding master Lagrangian is

$$
L_{M}^{(4)}=\frac{1}{8} \bar{T}^{[\mu \nu] \alpha} \bar{T}_{[\mu \nu] \alpha}+\frac{1}{4} \bar{T}^{[\mu \nu] \alpha} \bar{T}_{[\mu \alpha] \nu}-\frac{1}{2} \bar{T}^{\mu} \bar{T}_{\mu}+\frac{1}{4} \epsilon_{\mu \nu \alpha \beta}\left(\partial^{\nu} K^{[\alpha \beta] \sigma}\right) \phi_{\sigma}^{\mu}
$$

where $\bar{T}^{[\mu \nu] \alpha}=T^{[\mu \nu] \alpha}-K^{[\mu \nu] \alpha}$ and $\bar{T}^{\mu}=T^{\mu}-K^{\mu}$. As earlier, we have introduced here the gauge field $K_{[\mu \nu] \lambda}$ elevating the global shift invariance to a local one.

We start the dualisation from partition function

$$
Z=\int D \phi_{\alpha \beta} D e_{\mu \nu} D K_{[\sigma \rho] \lambda} e^{-i \int d^{4} x L_{M}^{(4)}}
$$

where the Lagrangian is given in equation (16). By varying $K^{[\mu \nu] \alpha}$ the equation of motion following is

$$
\begin{aligned}
& -\frac{1}{4} T^{[\mu \nu] \alpha}+\frac{1}{4} K^{[\mu \nu] \alpha}-\frac{1}{4} T^{[\mu \alpha] \nu}+\frac{1}{4} T^{[\nu \alpha] \mu}+\frac{1}{4} K^{[\mu \alpha] \nu}-\frac{1}{4} K^{[\nu \alpha] \mu} \\
& +\frac{1}{2} T^{\mu} g^{\nu \alpha}-\frac{1}{2} T^{\nu} g^{\mu \alpha}-\frac{1}{2} K^{\mu} g^{\nu \alpha}+\frac{1}{2} K^{\nu} g^{\mu \alpha}-\frac{1}{4} \epsilon^{\sigma \beta \mu \nu} \partial_{\beta} \phi_{\sigma}^{\alpha}=0 .
\end{aligned}
$$

From this we get

$$
\begin{aligned}
& \bar{T}^{\mu}=-\frac{1}{4} \epsilon^{\mu \beta \sigma \nu} F_{[\beta \sigma] \nu} \\
& \bar{T}^{[\mu \nu] \alpha}=\frac{1}{4}\left(g^{\alpha \mu} \epsilon^{\nu \beta \sigma \rho}-g^{\alpha \nu} \epsilon^{\mu \beta \sigma \rho}\right) F_{[\beta \sigma] \rho}-\frac{1}{2}\left(\epsilon^{\beta \sigma \alpha \mu} F_{[\beta \sigma]}^{\nu}-\epsilon^{\beta \sigma \alpha \nu} F_{[\beta \sigma]}^{\mu}\right) .
\end{aligned}
$$

Substituting this back in equation (17), which is equivalent to integrating out $K^{[\mu \nu] \alpha}$ and $e_{\mu \nu}$ we get the dual partition function

$$
Z=\int D \phi_{\mu \nu} e^{-i \int d^{4} x L_{D}^{(4)}}
$$

where $L_{D}^{(4)}$ is same as given in equation (15). Thus even directly from second order theory equation (2) one can arrive at the dual (second order) theory. The field content and the form of the dual theory is same as the original second order form equation (2). This is in agreement with earlier studies. This is similar to that of Maxwell theory in $3+1$ dimensions. This completes the dualisation of $4 \mathrm{~d}$ theory.

It should be noted that the technique adopted here to derive the dually equivalent formulation is different from Fradkin-Tseytlin [24]approach. In contrast to the FradkinTseytlin approach, the necessary condition in the present approach is the existence of a global symmetry in the original theory and is not restricted to a first-order formalism. Moreover, in this approach, at every stage gauge invariance of the original theory is preserved.

\section{Dual formulation in dimensions- $d>4$}

In this section we first study the dual construction in 5-dim. and then generalise it to arbitrary $d$ dimensions. The difference between 4-dim. and 5-dim. case comes in the term involving the multiplier field. This term, enforcing the flatness condition of the dual field strength now has 5-d Levi-Cività symbol and correspondingly, the multiplier 
field has a different tensorial structure compared to that in the 4-dim. case studied above.

In the present case, thus we start from the master Lagrangian

$$
\begin{aligned}
L_{M}^{(5)}= & -\frac{1}{2} \omega^{\mu[\nu \alpha]} \omega_{\nu[\mu \alpha]}+\frac{1}{2} \omega^{\mu} \omega_{\mu}-\frac{1}{2} \omega^{\mu[\nu \alpha]}\left(T_{[\nu \alpha] \mu}-K_{[\nu \alpha] \mu}\right) \\
& -\omega^{\nu} T_{\nu}+\omega^{\nu} K_{\nu}+\frac{1}{12} \epsilon^{\mu \nu \sigma \alpha \gamma}\left(\partial_{\mu} K_{[\nu \sigma] \beta}\right) \phi_{\alpha \gamma}{ }^{\beta} .
\end{aligned}
$$

Here the field strength is defined as $F_{[\mu \tau \gamma] \lambda}=\partial_{[\mu} \phi_{[\tau \gamma]] \lambda}$ and $\phi_{[\tau \gamma] \beta}$ field is mixed symmetric Lagrange multiplier field. As in 4-dim case, demanding $L_{M}^{(5)}$ to have the original spin-2 symmetry requires $\delta_{\Lambda} K_{[\nu \sigma] \beta}=0$ and $\delta_{\Lambda} \phi_{[\mu \nu] \lambda}=\frac{3}{2 !} \epsilon_{\mu \nu \lambda \rho \sigma} \Lambda^{[\rho \sigma]}(x)=\tilde{\Lambda}_{[\mu \nu \lambda]}$. The corresponding partition function is

$$
Z=\int D \phi_{[\alpha \beta] \lambda} D e_{\mu \nu} D \omega_{\sigma[\rho \lambda]} D K_{[\theta \tau] \lambda} e^{-i \int d^{5} x L_{M}^{(5)}} .
$$

The partition function of the dual theory is obtained by integrating over the gauge field $K_{[\mu \nu] \lambda}$, original fields $e_{\mu \nu}$ and $\omega_{\mu[\nu \lambda]}$ as in the 4-dim. The gauge transformation of the dual field, which is the multiplier field $\phi_{\alpha \gamma}{ }^{\beta}$, results from the arbitrariness in its definition in the master Lagrangian.

$L_{M}^{(5)}$ equation(21)is invariant under the following transformation(up to a total derivative)

$$
\phi_{\alpha \beta, \gamma} \rightarrow \phi_{\alpha \beta, \gamma}+\partial_{\alpha} \tilde{\epsilon}_{\beta \gamma}-\partial_{\beta} \tilde{\epsilon}_{\alpha \gamma}
$$

where $\tilde{\epsilon}_{\beta \gamma}$ is an arbitrary second rank tensor. The fields strength $F_{[\mu \tau \gamma] \lambda}$ is invariant under this transformation.

The $K_{[\mu \nu] \lambda}$ integrations leads to the delta function condition (as in the 4-dim) which now involve third rank mixed symmetric field and the $\omega_{[\mu \nu] \lambda}$ field and is given by

$$
\delta\left(\omega^{\beta[\nu \sigma]}+\left(\omega^{\nu} g^{\sigma \beta}-\omega^{\sigma} g^{\nu \beta}\right)+\frac{1}{6} \epsilon^{\mu \alpha \gamma \nu \sigma} F_{[\mu \alpha \gamma]}^{\beta}\right) .
$$

The partition function of the dual theory is obtained by integrating over $\omega_{\mu[\nu \lambda]}$ which can be done similar to $4 \mathrm{~d}$ case leading to

$$
Z=\int D \phi_{[\alpha \beta] \lambda} e^{-i \int d^{5} x L_{D}^{(5)}}
$$

where

$$
L_{D}^{(5)}=-\frac{1}{6} F_{[\mu \nu \gamma] \lambda} F^{[\mu \nu \gamma] \lambda}-\frac{1}{4} F_{[\mu \nu \gamma] \lambda} F^{[\mu \nu \lambda] \gamma}+\frac{3}{4} F_{[\mu \gamma \rho]}^{\rho} F_{\nu}^{[\mu \gamma \nu]} .
$$

The gauge symmetries of the dual Lagrangian are given by

$$
\begin{aligned}
\delta_{\tilde{\epsilon}} \phi_{\alpha \beta, \gamma} & =\partial_{\alpha} \tilde{\epsilon}_{\beta \gamma}-\partial_{\beta} \tilde{\epsilon}_{\alpha \gamma} \\
\delta_{\Lambda} \phi_{\alpha \beta, \gamma} & =\tilde{\Lambda}_{[\alpha \beta \gamma]} .
\end{aligned}
$$

These are the generalisation of equation(3) and equation (4) to $5 \mathrm{~d}$ in dual frame-like formulation. This is the same as the Lagrangian for spin-2 theory constructed in [11] using the $(2,1)$ tensor gauge field $\phi_{[\delta \gamma] \beta}$. 
In [13] dual form of FP theory in 5-dim. was shown to described by the Lagrangian

$$
\mathcal{L}=-\frac{1}{6}\left(F_{[\mu \nu \lambda] \gamma} F^{[\mu \nu \lambda] \gamma}-3 F_{[\mu \nu \lambda]}^{\lambda} F_{\rho}^{[\mu \nu \rho]}\right) .
$$

Here also $F_{[\mu \nu \lambda] \gamma}=\partial_{[\mu} \phi_{\nu \lambda] \gamma}$ but the fields obey the condition $\phi_{[[\mu \nu] \lambda]}=0$ which is absent in the dual theory derived in equation (26). Hence the question arises as to how equation (26) is related to equation (29) as both are dual to FP theory in $5 \mathrm{dim}$.

The Lagrangian in equation (29) is in metric-like formulation as the field satisfies $\phi_{[[\mu \nu] \lambda]}=0$, unlike the one given in equation (26) which is in frame-like formulation. The connection between them can be seen by working in the gauge $\phi_{[[\mu \nu] \lambda]}=0$, using equation (28). Then the gauge transformation in the equation (27), which preserves this gauge, is

$$
\delta \phi_{[\nu \gamma] \lambda}=\frac{1}{3}\left[\partial_{\nu} A_{[\gamma \lambda]}+\partial_{\gamma} A_{[\lambda \nu]}-2 \partial_{\lambda} A_{[\gamma \nu]}\right]+\partial_{[\nu} S_{\gamma] \lambda}
$$

where $A_{\alpha \beta}=-A_{\beta \alpha}$ and $S_{\alpha \beta}=S_{\beta \alpha}$. Note that the most general Lagrangian involving $F_{[\mu \nu \lambda] \rho}$ is

$$
L=-\left(F_{[\mu \nu \gamma] \lambda} F^{[\mu \nu \gamma] \lambda}+B F_{[\mu \nu \gamma] \lambda} F^{[\mu \nu \lambda] \gamma}+C F_{\mu \gamma \lambda}{ }^{\lambda} F_{\nu}^{\mu \gamma \nu}\right) .
$$

$F_{[\mu \nu \gamma] \lambda}$ is invariant under $S_{\alpha \beta}$ and by demanding the symmetry of (31) under $A_{[\gamma \lambda]}$ given in equation (30) gives the condition $3+B+C=0$. Thus there is a one-parameter of theories and for a choice of $\mathrm{B}=0, \mathrm{C}$ takes -3 which is Curtright's Lagrangian.

Now extending this procedure to arbitrary dimensions is straight forward. The starting Lagrangian is

$$
\begin{aligned}
L_{M}^{(d)} & =-\frac{1}{2} \omega^{\mu[\nu \alpha]} \omega_{\nu[\mu \alpha]}+\frac{1}{2} \omega^{\mu} \omega_{\mu}-\frac{1}{2} \omega^{\mu[\nu \alpha]}\left(T_{[\nu \alpha] \mu}-K_{[\nu \alpha] \mu}\right) \\
& -\omega^{\nu} T_{\nu}+\omega^{\nu} K_{\nu}+\frac{1}{2(d-2) !} \epsilon^{\mu_{1} \mu_{2} \mu_{3} \mu_{4} \cdots \mu_{d}} \partial_{\mu_{1}} K_{\left[\mu_{2} \mu_{3}\right] \beta} \phi_{\left[\mu_{4} \cdots \mu_{d}\right]}{ }^{\beta} .
\end{aligned}
$$

Here also in order to maintain the original spin-2 symmetry, the gauge field and multiplier field undergoes the corresponding transformations, $\delta_{\Lambda} K_{\left[\mu_{2} \mu_{3}\right] \beta}=0$ and $\delta_{\Lambda} \phi_{\left[\mu_{1} \mu_{2} \cdots \mu_{d-3}\right] \nu}=\frac{(d-2)}{2 !} \epsilon_{\mu_{1} \mu_{2} \cdots \mu_{d-3} \nu \sigma \lambda} \Lambda^{\sigma \lambda}(x)=\tilde{\Lambda}_{\left[\mu_{1}, \mu_{2} \cdots \mu_{d-3} \nu\right]}$.

The gauge freedom associated with the dual field ( i.e, multiplier field) owes to the arbitrariness in its definition in the action for $L_{M}^{(d)}$ by

$$
\delta_{\tilde{\epsilon}} \phi_{\left[\mu_{1} \mu_{2} \cdots \mu_{d-3}\right] \nu}=\partial_{\left[\mu_{1}\right.} \tilde{\epsilon}_{\left.\mu_{2} \cdots \mu_{d-3}\right] \nu}
$$

where $\tilde{\epsilon}_{\mu_{1} \cdots \mu_{d-4} \nu}$ is antisymmetric in first $\mu_{1}$ to $\mu_{d-4}$ indices.

Now after integrating out the gauge field $K_{\left[\mu_{1} \mu_{2}\right] \beta}$ and the original fields $e_{\mu \nu}, \omega_{\mu[\nu \lambda]}$ as in 4-dim. and 5-dim. we get the dual Lagrangian in frame-like field as

$$
\begin{aligned}
L_{D}= & \frac{1}{2(d-2)(d-2) !^{2}} \epsilon^{\mu_{1} \mu_{2} \mu_{3} \mu_{4} \cdots \mu_{d}} \epsilon_{\nu_{1} \mu_{2} \nu_{3} \nu_{4} \cdots \nu_{d}} F_{\left[\mu_{1} \mu_{4} \cdots \mu_{d}\right] \mu_{3}} F^{\left[\nu_{1} \nu_{4} \cdots \nu_{d}\right] \nu_{3}} \\
& -\frac{1}{2(d-2) !^{2}} \epsilon^{\mu_{1} \mu_{2} \mu_{3} \mu_{4} \cdots \mu_{d}} F_{\left[\mu_{1} \mu_{4} \cdots \mu_{d}\right]}{ }^{\nu_{2}} \epsilon_{\nu_{1} \nu_{2} \mu_{3} \nu_{4} \cdots \nu_{d}} F^{\left[\nu_{1} \nu_{4} \cdots \nu_{d}\right]}{ }_{\mu_{2}}
\end{aligned}
$$


where the field strength $F_{\mu_{1} \cdots \mu_{d-2}, \nu}$ is $\partial_{\left[\mu_{1}\right.} \phi_{\left.\mu_{2} \cdots \mu_{d-2}\right] \nu}$ and is invariant under $\tilde{\epsilon}$ transformation in equation (33). The symmetries of this dual Lagrangian are

$$
\begin{aligned}
\delta_{\tilde{\epsilon}} \phi_{\left[\mu_{1} \mu_{2} \cdots \mu_{d-3}\right] \nu} & =\partial_{\left[\mu_{1}\right.} \tilde{\epsilon}_{\left.\mu_{2} \cdots \mu_{d-3}\right] \nu} \\
\delta_{\Lambda} \phi_{\left[\mu_{1} \mu_{2} \cdots \mu_{d-3}\right] \nu} & =\tilde{\Lambda}_{\left[\mu_{1} \mu_{2} \cdots \mu_{d-3} \nu\right]}
\end{aligned}
$$

This agrees with equation (15) and equation (26) for $d=4$ and $d=5$ respectively. Also note that for $d=6$, the dual Lagrangian obtained here is same as the Lagrangian for $\phi_{\left[\mu_{1} \mu_{2} \mu_{3}\right] \mu_{4}}$ field constructed in [11]. Thus we get the general Lagrangian extending Zinoviev construction to d-dimensions and its identification as dual spin-2 theory follows from equation (34). The above Lagrangian can be written (apart from an overall factor) in a more convenient form as

$$
\begin{gathered}
L_{D}=(-1)^{d}\left[F_{\left[\mu_{1} \mu_{2} \cdots \mu_{d-2}\right] \nu} F^{\left[\mu_{1} \mu_{2} \cdots \mu_{d-2}\right] \nu}+\frac{(d-2)}{(d-3)} F_{\left[\mu_{1} \mu_{2} \cdots \mu_{d-2}\right] \nu} F^{\left[\mu_{1} \mu_{2} \cdots \mu_{d-3} \nu\right] \mu_{d-2}}\right. \\
\left.-\frac{(d-2)^{2}}{(d-3)} F_{\left[\mu_{1} \mu_{2} \cdots \mu_{d-3} \alpha\right]}^{\alpha} F^{\left[\mu_{1} \mu_{2} \cdots \mu_{d-3} \rho\right]}\right] .
\end{gathered}
$$

This form of the dual Lagrangian in a frame-like formulation is an important result of this work.

This form of Lagrangian can be argued from the gauge invariance of the dual theory. The most general Lagrangian involving the field strength $F_{\left[\mu_{1} \mu_{2} \cdots \mu_{d-2}\right] \nu}$ (which is invariant under $\tilde{\epsilon}$ transformation given in equation (35)) is of the form

$$
\begin{aligned}
L_{D}=(-1)^{d}[ & F_{\left[\mu_{1} \mu_{2} \cdots \mu_{d-2}\right] \nu} F^{\left[\mu_{1} \mu_{2} \cdots \mu_{d-2}\right] \nu}+B F_{\left[\mu_{1} \mu_{2} \cdots \mu_{d-2}\right] \nu} F^{\left[\mu_{1} \mu_{2} \cdots \mu_{d-3} \nu\right] \mu_{d-2}} \\
& \left.+C F_{\left[\mu_{1} \mu_{2} \cdots \mu_{d-3} \alpha\right]}^{\alpha} F_{\left[\mu_{1} \mu_{2} \cdots \mu_{d-3} \rho\right]} \rho\right]
\end{aligned}
$$

and by demanding the invariance given in equation (35) under $\tilde{\Lambda}$, the parameters get fixed as

$$
B=\frac{(d-2)}{d-3}, \quad C=-\frac{(d-2)^{2}}{(d-3)} .
$$

A metric-like formulation of Curtright's theory has been formulated in [25]. The connection of our frame-like formulation to the metric-like formulation can be obtained by fixing the gauge $\phi_{\left[\left[\mu_{1} \mu_{2} \cdots \mu_{d-3}\right] \nu\right]}=0$ using $\tilde{\Lambda}$ transformation in (35). Then the gauge transformation, which preserves this gauge under $\tilde{\epsilon}$ becomes

$$
\begin{gathered}
\delta \phi_{\left[\mu_{1} \mu_{2} \cdots \mu_{d-3}\right] \nu}=\partial_{\mu_{1}} A_{\mu_{2} \cdots \mu_{d-3} \nu}+\partial_{\mu_{2}} A_{\mu_{3} \cdots \mu_{d-3} \nu \mu_{1}}+\cdots-(d-3) \partial_{\nu} A_{\mu_{1} \mu_{2} \cdots \mu_{d-3}}+ \\
\partial_{\left[\mu_{1}\right.} \widehat{e}_{\left.\mu_{2} \cdots \mu_{d-3}\right] \nu}
\end{gathered}
$$

where A is totally antisymmetric tensor and $\widehat{e}_{\left[\mu_{2} \cdots \mu_{d-3}\right] \nu}$ is a mixed symmetric tensor with totally antisymmetric part removed. The field strength is invariant under $\widehat{e}$ parameter and invariance of equation (37) under A parameter gives a relation between the coefficients as $(d-2)+B+C=0$. Thus there is a one-parameter family of theories in metric-like formulation. 


\section{Dual generating functional in $d \geq 4$}

In this section we obtain the equivalence between the generating functionals and derive the mapping between the observables of the equivalent theories. For this we couple the original field to an appropriate source and it is natural to couple $e_{\mu \nu} J^{\mu \nu}$. But this does not have the shift symmetry of the field. The source has to couple to a combination of field which has the global shift symmetry and also all the other gauge symmetries of the original Lagrangian. Since we wish to use first order Lagrangian, the relevant gauge transformations are that given in equation (6) and we couple the gauge invariant observable $\partial_{\mu} \omega_{\nu[\alpha \beta]}-\partial_{\nu} \omega_{\mu[\alpha \beta]}$ to the source $J_{[\mu \nu][\alpha \beta]}$. The starting point now is the Lagrangian

$$
L_{J}^{(4)}=L_{I}+J_{[\mu \nu][\alpha \beta]}\left(\partial_{\mu} \omega_{\nu[\alpha \beta]}-\partial_{\nu} \omega_{\mu[\alpha \beta]}\right)
$$

where $L_{I}$ is equation (5). The gauge invariant observable chosen is linear Riemann curvature tensor. First we illustrate the operator correspondence in $4 \mathrm{~d}$ case. Now note that global shift symmetry in equation(5) not modified. Hence the master Lagrangian with the present augmentation of source $J_{[\mu \nu][\alpha \beta]}$ coupling is

$$
L_{M}^{(4)}(J)=L_{M}^{(4)}+J_{[\mu \nu][\alpha \beta]}\left(\partial^{\mu} \omega^{\nu[\alpha \beta]}-\partial^{\nu} \omega^{\mu[\alpha \beta]}\right)
$$

where $L_{M}^{(4)}$ is given in equation (9). Integrating over $\mathrm{K}$ field from the partition function corresponding to the above Lagrangian $L_{M}^{(4)}(J)$ gives the same delta function given in (12). Integrating over fields $\omega_{\mu[\nu \lambda]}$ and $e_{\mu \nu}$ result in the (dual) partition function corresponding to the (dual) Lagrangian

$$
\begin{aligned}
L_{D}^{(4)}[J] & =L_{D}^{(4)}-\frac{1}{2} J_{[\mu \nu][\alpha \beta]} \epsilon^{\alpha \beta \rho \lambda}\left(\partial^{\mu} \partial_{\rho} \phi_{\lambda}{ }^{\nu}-\partial^{\nu} \partial_{\rho} \phi_{\lambda}{ }^{\mu}\right) \\
& +\frac{1}{4} J_{[\mu \nu][\alpha \beta]}\left\{\partial^{\mu}\left(g^{\nu \beta} \epsilon^{\alpha \rho \lambda \gamma} \partial_{\rho} \phi_{\lambda \gamma}-g^{\nu \alpha} \epsilon^{\beta \rho \lambda \gamma} \partial_{\rho} \phi_{\lambda \gamma}\right)\right. \\
& \left.-\partial^{\nu}\left(g^{\mu \beta} \epsilon^{\alpha \rho \lambda \gamma} \partial_{\rho} \phi_{\lambda \gamma}-g^{\mu \alpha} \epsilon^{\beta \rho \lambda \gamma} \partial_{\rho} \phi_{\lambda \gamma}\right)\right\}
\end{aligned}
$$

where $L_{D}^{(4)}$ is given in equation (15). Note here that the terms coupled with source in above equation (42) has $\delta \phi_{\mu \nu}=\tilde{\Lambda}_{[\mu \nu]}(x)$ symmetry. Using this symmetry, the antisymmetric part of $\phi_{\mu \nu}$ can be gauge fixed to zero retaining only the symmetric part $\phi_{\mu \nu}^{s}$. Then the terms in the second and third line of equation (42) involving trace of $J_{[\mu \nu][\alpha \beta]}$ vanish. Non-vanishing source coupling terms is $-\frac{1}{2} J_{[\mu \nu][\alpha \beta]} \epsilon^{\rho \alpha \beta \lambda}\left(\partial^{\mu} \partial_{\rho} \phi_{\lambda}{ }^{\nu}-\right.$ $\left.\partial^{\nu} \partial_{\rho} \phi_{\lambda}{ }^{\mu}\right) \equiv-J \tilde{R}_{(L)}\left(\Phi^{s}\right)$ where $\tilde{R}_{(L)}\left(\Phi^{s}\right)$ is dual linear Riemann tensor. Usually in the mapping between the n-point correlators, dually equivalent n-point functions gets contributions from contact terms. Here similar terms can be seen to arise if in the partition function of the starting first-order theory equation (5), the $\omega_{\mu[\alpha \beta]}$ field integrals are done. The resulting second-order theory is of the form

$$
\begin{aligned}
L(J)= & L-J_{[\mu \nu][\alpha \beta]} \partial^{\nu}\left(T^{[\mu \alpha] \beta}-T^{[\mu \beta] \alpha}-T^{[\alpha \beta] \mu}\right) \\
& -30 \partial_{\nu} J^{[\mu \nu][\alpha \beta]} \partial^{\rho} J_{[\mu \rho][\alpha \beta]}+72 \partial^{\nu} J_{[\alpha \nu][\beta \mu]} g^{\alpha \beta} \partial_{\rho} J^{[\sigma \rho][\gamma \mu]} g_{\sigma \gamma}
\end{aligned}
$$

where $L$ is given in equation (2). Notice that the term linearly coupling to $J_{[\mu \nu][\alpha \beta]}$ can be identified as the Riemann tensor when we use the invariance of $e_{\mu \nu}$ field under 
equation (4). Apart from contact term, by functionally differentiating the partition functions corresponding to the Lagrangians in equation (41) and equation (42)with the source, we get

$$
<R_{\alpha \beta \mu \nu}(x) R_{\sigma \rho \gamma \delta}(y)>=<\tilde{R}_{\alpha \beta \mu \nu}(x) \tilde{R}_{\sigma \rho \gamma \delta}(y)>_{d u a l}
$$

where $\tilde{R}_{\alpha \beta \mu \nu}=\epsilon_{\alpha \beta \rho \sigma} R_{\rho \sigma \mu \nu}$.

Thus there is a duality mapping $R_{\alpha \beta \mu \nu}(h) \rightarrow \epsilon_{\mu \nu \rho \sigma} R^{\rho \sigma, \alpha \beta}\left(\Phi^{s}\right)$. Next we obtain the map in 5-dim. Proceeding in the same way one will have the dual Lagrangian with source coupled

$$
\begin{aligned}
L_{D}^{(5)}(J) & =L_{D}^{(5)}-\frac{1}{18} J_{[\mu \nu][\alpha \beta]}\left\{\partial^{\mu}\left(g^{\nu \beta} \epsilon^{\alpha \rho \tau \gamma \lambda} F_{[\rho \tau \gamma] \lambda}-g^{\nu \alpha} \epsilon^{\beta \rho \tau \gamma \lambda} F_{[\rho \tau \gamma] \lambda}\right)\right. \\
& \left.-\partial^{\nu}\left(g^{\mu \beta} \epsilon^{\alpha \rho \tau \gamma \lambda} F_{[\rho \tau \gamma] \lambda}-g^{\mu \alpha} \epsilon^{\beta \rho \lambda \tau \gamma \lambda} F_{[\rho \tau \gamma] \lambda}\right)\right\} \\
& -\frac{1}{6} J_{[\mu \nu][\alpha \beta]} \epsilon^{\alpha \beta \rho \tau \gamma}\left(\partial^{\mu} F_{[\rho \tau \gamma]}^{\nu}-\partial^{\nu} F_{[\rho \tau \gamma]}^{\mu}\right)
\end{aligned}
$$

where $L_{D}^{(5)}$ is given in equation (26). Now this $L_{D}^{(5)}$ also has $\delta \phi_{\left[\mu_{1} \mu_{2}\right] \mu_{3}}=\tilde{\Lambda}_{\left[\mu_{1} \mu_{2} \mu_{3}\right]}(x)$ invariance and using this (as in 4-d) terms involving trace of $J_{[\mu \nu][\alpha \beta]}$ can be set to vanish. From this we can identify the (linear) Riemann tensor as a function of dual field as $\left(\partial^{\mu} F_{\rho \tau \gamma}{ }^{\nu}-\partial^{\nu} F_{\rho \tau \gamma}{ }^{\mu}\right)$. As in the earlier case, we get the mapping $R_{\mu \nu, \alpha \beta} \rightarrow \epsilon_{\alpha \beta \rho \tau \gamma}\left(\partial_{\mu} F_{\nu}^{[\rho \tau \gamma]}-\partial_{\nu} F_{\mu}^{[\rho \tau \gamma]}\right)$. This is easily generalised to d-dimensions where the dual Lagrangian with source coupling is

$$
\begin{gathered}
L_{D}^{(d)}(J)=L_{D}^{(d)}-\frac{1}{(d-2) !} J_{[\mu \nu][\alpha \beta]} \epsilon^{\mu_{1} \alpha \beta \mu_{4} \cdots \mu_{d}}\left(\partial^{\mu} F_{\left[\mu_{1} \mu_{4} \cdots \mu_{d}\right]}^{\nu}-\partial^{\nu} F_{\left[\mu_{1} \mu_{4} \cdots \mu_{d}\right]}{ }^{\mu}\right) \\
+\frac{J_{[\mu \nu][\alpha \beta]}}{(d-2)(d-2) !}\left\{\partial^{\mu}\left(\epsilon^{\mu_{1} \alpha \mu_{3} \mu_{4} \cdots \mu_{d}} F_{\left[\mu_{1} \mu_{4} \cdots \mu_{d}\right] \mu_{3}} g^{\beta \nu}-\epsilon^{\mu_{1} \beta \mu_{3} \mu_{4} \cdots \mu_{d}} F_{\left[\mu_{1} \mu_{4} \cdots \mu_{d}\right] \mu_{3}} g^{\alpha \nu}\right)\right. \\
\left.-\partial^{\nu}\left(\epsilon^{\mu_{1} \alpha \mu_{3} \mu_{4} \cdots \mu_{d}} F_{\left[\mu_{1} \mu_{4} \cdots \mu_{d}\right] \mu_{3}} g^{\beta \mu}-\epsilon^{\mu_{1} \beta \mu_{3} \mu_{4} \cdots \mu_{d}} F_{\left[\mu_{1} \mu_{4} \cdots \mu_{d}\right] \mu_{3}} g^{\alpha \mu}\right)\right\}
\end{gathered}
$$

where $L_{D}^{(d)}$ is given in equation (34). Here we have identified $\frac{1}{(d-2) !}\left(\partial^{\mu} F_{\left[\mu_{1} \mu_{4} \cdots \mu_{d}\right]}{ }^{\nu}-\right.$ $\left.\partial^{\nu} F_{\left[\mu_{1} \mu_{4} \cdots \mu_{d}\right]}{ }^{\mu}\right)$ as the dual (linearised) Riemann tensor in terms of field strength. Thus we obtain a dual formulation of generating functional with source coupled to linear Riemann tensor, providing a mapping between Riemann tensor and dual Riemann tensor

$$
R^{\mu \nu \alpha \beta} \rightarrow \epsilon^{\alpha \beta \mu_{1} \mu_{4} \cdots \mu_{d}} \mathcal{R}_{\mu_{1} \mu_{4} \cdots \mu_{d}}{ }^{\nu \mu}
$$

where the Riemann curvature tensor for the dual field $\Phi_{\mu_{4} \mu_{5} \ldots \mu_{d}}{ }^{\nu}$ is

$$
\mathcal{R}_{\mu_{1} \mu_{4} \cdots \mu_{d}}^{\nu \mu}=\left(\partial^{\mu} F_{\mu_{1} \mu_{4} \cdots \mu_{d}}^{\nu}-\partial^{\nu} F_{\mu_{1} \mu_{4} \cdots \mu_{d}}{ }^{\mu}\right) .
$$

\section{Conclusion}

In this paper we have constructed the dual linearised gravity theory in arbitrary dimensions in frame-like formulation. We start with a frame-like formulation of linearised gravity, in a form equivalent to Fierz-Pauli theory and obtain the dual theory using the global shift of tetrad field. Using this method we have obtained the partition function in frame like formulation for dual theory in arbitrary dimensions. The dual theory obtained in 5-dim. and 6-dim coincide with the theory considered by Zinoviev 
for mixed symmetric tensor gauge fields $\phi_{[\mu \nu] \lambda}$ and $\phi_{[\mu \nu \lambda] \sigma}$ respectively. We have given the dual theory in arbitrary dimensions in a closed form (up to an over all factor) as a sum of three terms. The relative coefficients are crucial for the Lagrangian to have the required invariance of dual spin-2 theory. Next we extend this to provide equivalence at the level of n-point correlators, by coupling source to gauge invariant observable, the (linear) Riemann tensor. This gives Riemann tensor in terms of the dual gravitational field in d-dimensions. We thus show that the dual $\mathrm{s}=2$ theory can be obtained at the level of partition function and generating functional within the standard dual procedure.

We can also see that duality mapping derived here between the Riemann tensor for

the $h_{\mu \nu}$ field and that of the dual field implies the duality in the sense of exchanging Bianchi identity and equation of motion. The details are in the appendix.

Although we have considered the dual formulation only at quadratic level (noninteracting), it is desirable to extend this to cubic and higher order (interacting) terms. There are several arguments indicating that duality symmetry must exist at interacting case also. Hence the free-field analysis carried out here in a constructive scheme should be considered as a first step towards the more fundamental problem of constructing dual gravity including non-linearities. But in recent times several no-go theorems has been proved [26], showing dual formulation of non-linear gravity should fall beyond the scope of conventional perturbative local field theory.

\section{Acknowledgments}

EH thanks FAPESP for support through grant 03/09044-9. MS thanks Associateship Scheme for visit to The Abdus Salam ICTP, where part of the work was done and acknowledges discussion with L. Alvarez-Gaume. MS acknowledges DST (India)for support through a project. We thank the referees for several useful suggestions.

\section{Appendix A. Duality between the equation of motion and Bianchi Identity}

In this appendix we show that duality mapping derived between curvature tensor in terms of $h_{\mu \nu}$ and the dual field implies duality exchange between equation of motion and Bianchi identity. Using the duality map in equation (47) we first re-express the Ricci tensor $R_{\mu \nu}$ in terms of the dual field as

$$
\begin{aligned}
R_{\mu \nu} & =\epsilon_{\alpha \nu \rho_{3} \ldots \rho_{d}}\left(\partial^{\alpha} F^{\left[\rho_{3} \ldots \rho_{d}\right]}{ }_{\mu}-\partial_{\mu} F^{\left[\rho_{3} \ldots \rho_{d}\right] \alpha}\right) \\
& =-\epsilon_{\alpha \nu \rho_{3} \ldots \rho_{d}} \mathcal{R}^{\left[\rho_{3} \ldots \rho_{d}\right] \alpha}{ }_{\mu} .
\end{aligned}
$$

Note here that the Ricci tensor of $h_{\mu \nu}$ is thus mapped to the Bianchi identity of the Riemann curvature tensor corresponding to the dual field. Using this, we see that the equation of motion of (sourceless) linear gravity (in terms of $h_{\mu \nu}$ field) becomes

$$
R_{\mu \nu}=0 \longrightarrow \epsilon_{\alpha \nu \rho_{3} \ldots \rho_{d}} \mathcal{R}^{\rho_{3} \ldots \rho_{d} \alpha}{ }_{\mu}=0
$$

showing that the equation of motion for $h_{\mu \nu}$ gets mapped to the Bianchi identity for the dual curvature under the map derived in equation (47). Under this duality map in 
equation (47), the Bianchi identity $R_{[\alpha \beta \mu] \nu}=0$ becomes

$$
\begin{aligned}
R_{[\alpha \beta \mu] \nu}=0 & \longrightarrow \epsilon_{\alpha \beta \mu \sigma_{4} \ldots \sigma_{d}} \epsilon^{\mu \nu \rho_{3} \ldots \rho_{d}}\left(\partial^{\alpha} F_{\rho_{3} \ldots \rho_{d}}{ }^{\beta}-\partial^{\beta} F_{\rho_{3} \ldots \rho_{d}}{ }^{\alpha}\right) \\
& =\epsilon_{\alpha \beta \mu \sigma_{4} \ldots \sigma_{d}} \epsilon^{\mu \nu \rho_{3} \ldots \rho_{d}} \mathcal{R}_{\rho_{3} \ldots \rho_{d}} \beta=0 .
\end{aligned}
$$

Now the equation of motion following from the Lagrangian in equation (36) is

$2 \partial^{\mu_{1}} F_{\left[\mu_{1} \mu_{2} \ldots \mu_{d-2}\right] \nu}+\frac{1}{(d-3)} \partial^{\mu_{1}} F_{\left[\mu_{1} \mu_{2} \ldots \mu_{d-3}|\nu| \mu_{d-2}\right]}-\frac{(d-2)}{(d-3)} \partial^{\mu_{1}} F_{\left[\mu_{1} \mu_{2} \ldots \mu_{d-3} \mid \alpha\right.}^{\alpha \mid} g_{\left.\mu_{d-2}\right] \nu}=0$ (A.4)

Since the dual map in equation (47) is derived, only after gauge symmetry $\delta \phi_{\Lambda}=$ $\tilde{\Lambda}_{\mu_{1} \ldots \mu_{d-3}, \nu}$ is fixed, we have to do the same for the above equation of motion also. With this the equation (A.4) becomes

$$
-\partial^{\mu_{1}} F_{\left[\mu_{1} \mu_{2} \ldots \mu_{d-2}\right] \nu}+\partial^{\mu_{1}} F_{\left[\mu_{1} \mu_{2} \ldots \mu_{d-3} \mid \alpha\right.}^{\alpha \mid} g_{\left.\mu_{d-2}\right] \nu}=0
$$

which is identical to the right hand side of equation (A.3). Thus we see that the Bianchi identity of linear gravity goes to the equation of motion under the duality map.

Using the duality map in equation (47) the other Bianchi identity $\partial_{[\mu} R_{\nu \rho] \alpha \beta}=0$ becomes

$$
\epsilon_{\mu \nu \rho \sigma_{4} \ldots \sigma_{d}} \partial^{\mu}\left(\epsilon^{\alpha \beta \rho_{3} \ldots \rho_{d}}\left[\partial^{\nu} F_{\rho_{3} \ldots \rho_{d}}{ }^{\rho}-\nu \leftrightarrow \rho\right]\right)=0
$$

which is satisfied identically showing that the dual map is consistent with the Bianchi identity.

\section{References}

[1] Savit R 1980 Duality in field theory and statistical systems Rev. Mod. Phys. 52453 Olive D I 1997 Exact electromagnetic duality Nucl. Phys. B58 (Proc. Suppl) 43 Harvey J Magnetic monopoles, duality and supersymmetry (Preprint hep-th/9603086)

[2] García-Compean H, Obregón O, Plebañsky J F and Ramírez C 1998 Towards a gravitational analogue to S duality in non-Abelian gauge theories, Phys. Rev. D57 7501

García-Compean H, Obregón O and Ramírez C, 1998 Gravitational duality in MacDowell-Mansouri gauge theory Phys. Rev. D58 104012

[3] Nieto J A 1999 S-Duality for Linearized Gravity Phys. Lett A262 274

[4] Casini H, Montemayor R and Urrutia L F 2001 Dual theories for mixed symmetry fields. Spin-two case: $(1,1)$ versus $(2,1)$ Young symmetry type fields Phys. Lett. B507 336

[5] Hull C 2001 Duality in gravity and higher spin gauge fields JHEP 019027

[6] Medeiros P de and Hull C 2003 Exotic tensor gauge theory and duality Commun. Math. Phy. 235 255

[7] Casini H, Montemayor R and Urrutia L F 2003 Duality for symmetric second rank tensors. II. The linearized gravitational field Phys.Rev. D68 065011

[8] Bekaert X and Boulanger N 2004 Tensor gauge fields in arbitrary representations of GL(D, R): duality and Poincaré lemma Commun. Math. Phys. 24527

[9] Deser S, Townsend P K and Siegel W 1981 Higher rank representations of lower spin Nucl. Phys.B184 333

[10] Curtright T 1985 Generalized gauge fields Phy. Lett. B165 304

[11] Zinoviev Yu M First Order Formalism for Mixed Symmetry Tensor Fields (Preprint hepth/0304067)

[12] Vasiliev M A 1980 Gauge form of description of massless fields with arbitrary spin Fiz Yad. 32 855 (In Russian) 
[13] Boulanger N, Cnockaert S and Henneaux M 2003 A note on spin-s duality JHEP 0306060

[14] Matveev A S and Vasiliev M A 2005 On dual formulation for higher spin gauge fields in $(A) d S_{d}$ Phys. Lett. B609 157

[15] Henneaux M and Teitelboim C 2005 Duality in linearized gravity Phys. Rev. D71 024018

[16] Zinoviev Yu M On dual formulation of Gravity (Preprint hep-th/0504210)

[17] Lindström U and Rocek M 1983 Scalar tensor duality and N =1,2 non-linear -models Nucl. Phys. B222 285

Hitchin N, Karlhede A, Lindström U and Rocek M 1987 Hyper-Kahler metrics and supersymmetry Commun. Math. Phys. 108535

Buscher T H 1987 A symmetry of string background field equation Phys. Lett. B194 59

Buscher T H 1988 Path-integral derivation of quantum duality in nonlinear sigma-models Phys. Lett. B201 466

[18] Alvarez E, Alvarez-Gaume L and Bakas LI 1996 Supersymmetry and Dualities Nucl. Phys. (Proc. Suppl.) $\mathbf{4 6} 16$

[19] Burgess C P and Quevedo F 1994 Bosonization as duality Nucl. Phys. B421 373

[20] Harikumar E and Sivakumar M 1998 Duality and massive gauge invariant theories Phy. Rev. D57 3794

[21] Balachandran A P, Chander L and Sathiapalan B 1996 Chern-Simon duality and the Quantum Hall Effect Int. J. Mod. Phy. A11 3587

[22] Hjelmeland S E and Lindström U Duality for the non-specialist (Preprint hep-th/9705122)

[23] Witten E 1995 On S duality in abelian gauge theory Selecta Math. 1383

[24] Fradkin E S and Tseytlin A A 1985 quantum equivalence of dual field theories Ann. Phys.162 31

[25] Aulakh C S, Koh I G and Ouvry S 1986 Higher spin fields with mixed symmetry Phys. Lett. B173 284

[26] Deser S and Seminara D 2005 Free Spin 2 Duality invariance cannot be extended to GR Phys. Rev. D71 081502

Xavier Bekaert, Nicolas Boulanger and Sandrine Cnockaert 2005 No Self-Interaction for TwoColumn Massless Fields J. Math. Phys. 46012303

Xavier Bekaert, Nicolas Boulanger and Marc Henneaux 2003 Consistent deformations of dual formulations of linearized gravity: A no-go result Phys. Rev. D67 044010 